\title{
Rapid maxillary expansion treatment could produce long-term transverse skeletal changes
}

\author{
Are there any long-term (minimum of 1 year after active treatment) transverse, \\ anteroposterior and vertical skeletal changes after rapid maxillary expansion \\ treatment of subjects who have constricted arches?
}

\begin{abstract}
Lagravere MO, Major PW, Flores-Mir C. Long-term skeletal changes with rapid maxillary expansion: a systematic review. Angle Orthod 2005; 75:833-839
\end{abstract}

Data sources Medline, Medline In-Process and other Medline nonindexed citations, LILACS, Pubmed, Embase, Web of Science and the Cochrane Library were searched. Search terms were rapid palatal expansion or rapid maxillary expansion (RME), bone and bones or skeletal changes. Reference lists from retrieved articles were also examined.

Study selection For inclusion, an article had to satisfy the following criteria: describe controlled clinical trials; make measurements for facial radiographs (anteroposterior and lateral cephalograms); and include no surgical or simultaneous treatment that could alter RME effects during the evaluation period. Articles without an adequate control group to factor out normal growth changes were excluded.

Data extraction and synthesis Selected articles were independently evaluated by three researchers. Interexaminer discrepancies were resolved by discussion until a consensus was reached. A summary of sample size, retention period, measurement error and radiographs were listed in a table. A quality checklist of methods used was developed to evaluate the selected articles.

Results The search identified 188 articles, of which three met the inclusion criteria. From the final three articles, one measured transverse changes, two anteroposterior changes, and two vertical changes. The only statistically significant difference in skeletal width increase for subjects before and after the peak pubertal growth spurt was lateronasal width $(+1.5 \mathrm{~mm})$. For the early-treated group, the maxillary width increase was significant $(3 \mathrm{~mm})$, but not for the latetreated group $(0.9 \mathrm{~mm})$. Regarding anteroposterior changes in the maxilla and mandible, no significant alterations were found. Short-term and long-term vertical skeletal changes associated with RME appeared to be restricted to the maxilla. The magnitude of change was small and had little, if any, clinical significance.

Conclusions Long-term transverse skeletal maxillary increase is approximately $25 \%$ of the total dental expansion for prepubertal adolescents. Better long-term outcomes are expected in transverse changes because of RME in less skeletally mature patients. RME did not appear to produce clinically significant anteroposterior or vertical changes in the position of the maxilla and mandible. The conclusions from this systematic review should be considered with caution because only secondary-level evidence was found. Long-term randomised clinical trials (RCT) are needed.

\section{Commentary}

This study is the second part of a review of the long-term changes with RME by the same authors. The first part concerns itself mainly with dental changes and concludes that long-term transversal changes by RME are clinically significant. ${ }^{1}$

Although it is believed that RME produced changes primarily in the underlying skeletal structures rather than by the movement of teeth through alveolar bone, ${ }^{2,3}$ there have been no long-term studies on the skeletal changes by RME. This was aimed to be a systematic review of long-term skeletal changes after RME treatment.

After applying a similar search strategy to their first one, ${ }^{1}$ only three studies fulfilled the inclusion criteria. Even these three studies presented methodological problems such as a lack of description of a statistical estimation process for the sample size, dropouts and intra- and interexaminer reliability. Two of the three studies also figure in the first review on dental changes. This means that there are only five studies in all available literature that meet the inclusion criteria for a review of the long-term dental and skeletal changes after RME. The conclusions, as the authors stressed themselves, should thus be evaluated with caution: long-term RCT are required to obtain sound clinical conclusions about the effectiveness of RME at the skeletal level.

The authors do address an important issue: the relationship between scientific results and clinical significance and, further, with cost-effectiveness, the latter overlooked in many clinical studies.

\section{Yijin Ren}

Orthodontic Department, University of Gröningen, Gröningen, The Netherlands

1. Lagravere MO, Major PW, Flores-Mir C. Long-term dental arch changes after rapid maxillary expansion treatment: a systematic review. Angle Orthod 2005; 75: 151-157.

2. Wertz RA. Skeletal and dental changes accompanying rapid midpalatal suture opening. Am J Orthod 1970; 58:41-66.

3. McNamara JA. Early intervention in the transverse dimension: is it worth the effort? Am J Orthod Dentofacial Orthop 2002; 121:572-574.

Evidence-Based Dentistry (2005) 6, 92.

doi:10.1038/sj.ebd.6400359 